\title{
HIV-1 tropism and CD4 T lymphocyte recovery in a prospective cohort of patients initiating HAART in Ribeirão Preto, Brazil
}

\author{
Andre Minhoto Lanca', Jeova Keny Baima Collares², João Leandro de Paula Ferreira', \\ Danielle Malta Lima ${ }^{2}$, Luis Fernando de Macedo Brigido ${ }^{1 /+}$, \\ Rosangela Rodrigues ${ }^{1}$, Benedito Antonio Lopes da Fonseca ${ }^{3}$
}

\author{
${ }^{1}$ Laboratório de Retrovirus, Centro de Virologia, Instituto Adolfo Lutz, São Paulo, SP, Brasil ${ }^{2}$ Universidade de Fortaleza, \\ Fortaleza, CE, Brasil ${ }^{3}$ Centro de Pesquisa em Virologia, Faculdade de Medicina, Universidade de São Paulo, Ribeirão Preto, SP, Brasil
}

\begin{abstract}
While human immunodeficiency virus (HIV)-1 chemokine co-receptors 5 tropism and the GWGR motif in the envelope third variable region (V3 loop) have been associated with a slower disease progression, their influence on antiretroviral response remains unclear. The impact of baseline $V 3$ characteristics on treatment response was evaluated in a randomised, double blind, prospective cohort study with patients initiating highly active antiretroviral therapy with lopinavir or efavirenz plus azithothymidine/3TC (1:1) over 48 weeks. Similar virological and immunological responses were observed for both treatment regimens. The 43 individuals had a mean baseline CD4 T cell count of 119 cells $/ \mathrm{mm}^{3}$ [standard deviation $(S D)=99$ ] and a mean viral load of $5.09 \log _{10}$ copies $/ \mathrm{mL}(S D=0.49)$. The GWGR motif was not associated with a CD4 T cell response, but predicted R5 tropism by the geno2pheno [clinical20\%] $_{\text {algorithm }}$ correlated with higher CD4 T cell levels at all monitoring points $(p<0.05)$. Moreover, higher false-positive rates $(F P R)$ values from this analysis revealed a strong correlation with CD4 T cell recovery $(p<0.0001)$. Transmitted drug resistance mutations, documented in 3/41 (7.3\%) cases, were unrelated to the assigned antiretroviral regimen and had no impact on patient outcomes. In conclusion, naïve HIV-1 R5 infected patients exhibited higher CD4 T cell counts at baseline; this difference was sustained throughout therapy. The geno2pheno ${ }_{\text {[clinical] }}$ option FPR positively correlated with CD4 T cell gain and may be useful in predicting CD4 T cell recovery.
\end{abstract}

Key words: HIV-1 - disease progression - CD4 T lymphocytes - viral tropism - diversity - Brazil

Since the identification of human immunodeficiency virus (HIV)-1 as the aetiological agent of acquired immune deficiency syndrome (AIDS), several studies have associated different viral molecular characteristics, especially the diversity of the envelope gene, with disease progression (Potts et al. 1993, Santoro-Lopes et al. 2000). Most of these studies are focused on the envelope third variable region, or V3 loop, which is involved in HIV-1 binding to the chemokine co-receptors 5 (R5) and/or CXCR4 (X4). This step is necessary for viral fusion to the host cell (Shioda et al. 1991). A change in viral tropism has been associated with different phases of HIV-1 infection, with a predominance of R5 tropic virus observed at the initial stages of infection. X4-tropic viruses emerge at advanced disease stages in about half of all cases and this emergence is correlated with clinical progression and CD4 T cell depletion (Richman \& Bozzette 2004, Goetz et al. 2009). The pathogenic potential of $X 4$ virus is highlighted by its capacity to induce syncytia formation (Richman \& Bozzette 2004). Several bioinformatics tools have been used to determine viral genotypic

Financial support: FAPESP (2009/8215-0, 2006/61311-0), CNPq $(144076 / 2010-1)$

+ Corresponding author: lubrigido@gmail.com

Received 15 June 2011

Accepted 24 December 2011 tropism, such as the website geno2pheno and the 11/25 rule. Additionally, amino acid signatures at the V3 loop tip tetramer, which commonly harbour a proline at codon $16\left({ }_{15} \mathrm{GPGR}_{18}\right)$, show a substitution to tryptophan (W) at this position in some Brazilian clade $\mathrm{B}$ envelopes and are referred to as B' or Brazilian-B (Shioda et al. 1991, Potts et al. 1993, Pinto et al. 2008). The GWGR signature has a prevalence of $20-50 \%$ in Brazil and has been associated with a slower progression to AIDS (Santoro-Lopes et al. 2000, Casseb et al. 2002, 2004, de Brito et al. 2006). However, the actual relevance of this molecular diversity on treatment response is not yet clear. To expand the understanding of the potential role of genotypic viral tropism, false-positive rate (FPR) values obtained at geno2pheno and the presence of the GWGR motif in patients on antiretrovirals (ARV), the correlations of these characteristics with viral loads (VLs) and $\mathrm{CD} 4^{+} \mathrm{T}$ lymphocyte counts were assessed in patients during 48 weeks of firstline highly active antiretroviral therapy (HAART).

\section{PATIENTS, MATERIALS AND METHODS}

Study population - HIV-1-infected individuals not exposed to antiretroviral therapy (ART), but with indications for treatment according to the Brazilian Clinical Guideline, were invited and those eligible were randomly assigned in a 1:1 ratio to receive either lopinavir or efavirenz with azithothymidine (AZT)/3TC backbone. Clinical follow-up was conducted at the HIV Clinic, Medicine School Hospital, Ribeirão Preto, São Paulo University to evaluate the virological and immunological responses of the patients to HAART during the 48 weeks of follow- 
up. Data from patients who died before completing the follow-up were included in the baseline evaluation and the individuals who did not complete all visits were included in an intention to treat (ITT) analysis. A written informed consent was obtained from all patients and both Institutional Review Boards approved the study.

Laboratory and molecular analysis - CD4 T cell counts and percentages (flow cytometry, BD, USA) and VLs (Branched DNA, Siemens, USA) were determined at baseline and at weeks 6, 12, 24, 36 and 48. CD4 T cell gain was calculated as the absolute cell count from each week of observation minus the baseline CD4 T cell count. HIV-1 RNA and genomic DNA were extracted using the QIAamp ${ }^{\circledR}$ RNA/DNA extraction kit (Qiagen, Germany) according to manufacturer instructions and were stored at $-70^{\circ} \mathrm{C}$ until used. Synthesis of complementary DNA (cDNA) was performed by reverse transcription with Superscript III (Invitrogen, USA). Polymerase chain reaction amplification was performed on cDNA $(p o l)$ and proviral DNA (env) using a nested protocol as previously described (Ferreira et al. 2008). Amplicons were sequenced directly using BigDye and were resolved with an ABI 3100 Genetic Analyzer (Applied Biosystems, USA). Sequences of partial env and pol genes were manually edited with Sequencher 4.14 (GeneCodes, USA) software. Multiple sequence alignments were performed using the CLUSTALW algorithm with a reference set obtained from the Los Alamos Sequence Database. HIV subtyping was screened using NCBI Genotyping and was confirmed by phylogenetic methods using the software PAUP* version 4.0b10 (Sinauer Associates, USA). Transmitted drug resistance mutations (tDRM) were defined according to Calibrated Population Resistance v.6 (HIV Stanford Database) and the International AIDS Society (IAS) 2010 mutation list. Viral tropism was evaluated using the website geno2pheno ${ }_{\text {[co-receptor] }}$ bioinformatics tool (Sing et al. 2007) and both clonal and clinical options were used to obtain FPR values. Cut-offs of 5.75\% (MOTIVATE option) and 20\% (Vanderkerckhove et al. 2011) were employed to predict X4 tropism using clonal and clinical data. To obtain the FPR using the clinical option, baseline (absolute and percentage) CD4 T cells counts and VLs were added to the model. These predictions were analysed both as categorical variables (X4 or R5) and as continuous variables. V3 loop amino acid composition was determined by analysis of alignments with the HXB2 reference sequence from the Los Alamos Sequence Database (accession K03455).

Statistical analysis - Categorical variables were tested using Fischer's exact test or the Yates corrected test (two-tailed), as appropriate. Continuous variables were tested using the Mann-Whitney test, two-way analysis of variance (ANOVA) (non-parametric), the Spearman correlation and linear regression. Epinfo6 (Centers for Disease Control and Prevention) and GraphPad Prism 5.0 software were used in the statistical analysis. Virological and CD4 $\mathrm{T}$ cell outcomes were evaluated as ITT, missing equals failure (ITT, $\mathrm{M}=\mathrm{F}$ ) and as treated (AT), missing equals exclusion $(\mathrm{AT}, \mathrm{M}=\mathrm{E})$.

\section{RESULTS}

The 43 patients enrolled from 2004-2006 were mostly male $(70 \%)$ and had a mean age of 41 years [standard deviation (SD) 15 years], a mean baseline CD4 T cell count of 119 cells $/ \mathrm{mm}^{3}\left(\mathrm{SD}=99\right.$ cells $\left./ \mathrm{mm}^{3}\right)$ and a mean VL of $5.09 \log _{10} \mathrm{c} / \mathrm{mL}(\mathrm{SD}=0.49)$. CD4 T cell counts and VLs are depicted in Table. Three patients died and two abandoned therapy before their first return at week 6 of the follow-up. One patient discontinued treatment before 24 weeks and three left between 24-36 weeks of the follow-up. All three deaths occurred before concluding the clinical follow-up and these patients had baseline CD4 T lymphocyte counts below 50 cells $/ \mathrm{mm}^{3}$.

Patients were randomly assigned in a 1:1 ratio to receive either lopinavir (48.8\%) or efavirenz (51.2\%) with AZT/3TC backbone. The tDRM frequency at baseline (available after treatment initiation) was 7.3\%, all mutations were observed in the protease gene (mutations V82L, I85V and G73S) and the predicted loss of ART susceptibility was not relevant to the assigned ARV regimen. V82L and I85V substitutions were observed in patients assigned to receive lopinavir; these individuals exhibited a similar response to those without detectable mutations. The G73S mutation was observed in a patient receiving efavirenz who died two weeks after enrolment without an evaluation of the ARV response.

Virological and immunological outcomes after HAART on both regimen arms were comparable, with no differences between lopinavir and efavirenz response except for a higher occurrence of hypertriglyceridemia in the lopinavir group (data not shown). Viral suppression was achieved in most patients by week 24, when $89 \%$ of individuals had viremia below detection limits. After 48 weeks of HAART, only two individuals $(6 \%)$ had a detectable VL ( $>50$ copies $/ \mathrm{mL})$, with one on each regimen $\operatorname{arm}(\mathrm{AT}, \mathrm{M}=\mathrm{E})$. Further genotyping test analysis of one of these cases, RP16 on lopinavir + AZT/3TC therapy with several adherence issues, revealed the emergence of the mutation $\mathrm{M} 184 \mathrm{~V}$, which is predicted to reduce $3 \mathrm{TC}$ susceptibility.

$\mathrm{V} 3$ env region sequencing was performed for the 43 patient samples, with 42 sequences validated; one sequence for each sample was obtained. All HIV-1 envelopes were clade $\mathrm{B}$, except for one subtype $\mathrm{F}$ that was excluded from the Brazilian-B GWGR analysis. HIV-1 predicted tropism to CXCR4 co-receptor prevalence was $17 / 42(40 \%)$ when using geno2pheno ${ }_{[\text {clonal } 20 \%]}, 24 / 42$ (57\%) when using geno2pheno ${ }_{\text {[clinical20\%] }}$ and $6 / 42(14 \%)$ when using the MOTIVATE option (Table). Prediction of three sequences classified as X4 and 10 sequences classified as R5 by a clonal algorithm were discordant when clinical data were incorporated into the algorithm. When sequences were classified by the V3 loop motif, 4/9 (44\%) of GWGR, 4/12 (33\%) of GPGR and 9/20 (45\%) of "other

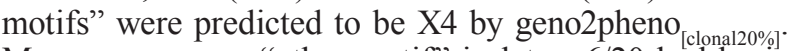
Moreover, among "other motif" isolates, 6/20 had basic amino acids at positions 11 or 25 and $3 / 20$ had a GWRR motif. No GPGR or GWGR isolate had basic amino acids at these positions. The six viruses harbouring basic amino acids at positions 11 and 25 had clonal and clinical FPR values below $20 \%$, except for one sequence. This case had 
TABLE

CD4 T cell counts and viral load (VL) according to highly active antiretroviral therapy (HAART) allocation group, V3 motif and predicted viral tropism

\begin{tabular}{|c|c|c|c|c|}
\hline & n $(\%)$ & $\begin{array}{c}\text { Baseline VL } \\
\text { mean (SD) }\end{array}$ & $\begin{array}{c}\text { Baseline CD4 T cell } \\
\text { mean (SD) }\end{array}$ & $\begin{array}{c}\text { CD4 T cells gain at } 48 \text { week } \\
\text { mean (SD) }\end{array}$ \\
\hline \multicolumn{5}{|l|}{ Randomization } \\
\hline Lopinavir + zidovudina /3TC & $21(49)$ & $5.09(0.12)$ & $117(22)$ & $223(22)$ \\
\hline Efavirenz + zidovudina /3TC & $22(51)$ & $5.09(0.09)$ & $122(21)$ & $212(29)$ \\
\hline \multicolumn{5}{|l|}{ V3 Motif } \\
\hline GPGR & $12(29.3)$ & $5.12(0.12)$ & $104(23)$ & $235(43)$ \\
\hline GWGR & $9(21.9)$ & $5.22(0.17)$ & $134(34)$ & $187(38)$ \\
\hline Other & $20(48.8)$ & $4.99(0.12)$ & $117(24)$ & $219(23)$ \\
\hline \multicolumn{5}{|l|}{ Tropism } \\
\hline \multicolumn{5}{|l|}{$11 / 25$ rule } \\
\hline $\mathrm{X} 4$ & $6(14.3)$ & $4.98(0.27)$ & $99(48)$ & $180(41)$ \\
\hline R5 & $36(85.7)$ & $5.11(0.08)$ & $125(17)$ & $223(21)$ \\
\hline \multicolumn{5}{|l|}{ MOTIVATE (5.7\%) } \\
\hline X4 & $6(14.3)$ & $5.13(0.19)$ & $70(31)$ & $183(53)$ \\
\hline R5 & $36(85.7)$ & $5.09(0.08)$ & $130(17)$ & $222(20)$ \\
\hline \multicolumn{5}{|l|}{ geno2pheno $_{\text {[clonall120\%] }}$} \\
\hline $\mathrm{X} 4$ & $17(40.5)$ & $5.07(0.15)$ & $90(23)$ & 187 (19) \\
\hline R5 & $25(59.5)$ & $5.11(0.08)$ & $142(20)$ & 237 (28) \\
\hline \multicolumn{5}{|l|}{ geno2pheno ${ }_{\text {[clinical } 20 \%]}$} \\
\hline $\mathrm{X} 4$ & $24(57.1)$ & $5.16(0.11)$ & $84(20)^{a}$ & 192 (18) \\
\hline R5 & $18(42.9)$ & $5.00(0.10)$ & $170(20)^{a}$ & $245(34)$ \\
\hline
\end{tabular}

$a: \mathrm{p}<0.01$. CD4 T (cells $\left./ \mathrm{mm}^{3}\right)$ and VL $\left(\log _{10}\right.$ copies $\left./ \mathrm{mL}\right)$ values at baseline and CD4 T cells gain at the last monitoring point (week 48) according to HAART group. Cases were pooled and described according to V3 loop motif and tropism prediction. Tropism was predicted using 11/25 rule and geno2pheno algorithm, considering a $20 \%$ cut-off as false-positive rate on clinical and clonal options and using MOTIVATE 5.75\% clonal cut-off. SD: standard deviation.

a $30 \%$ FPR value when we used the clinical algorithm, despite presenting an arginine $(\mathrm{R})$ at position 25 . The V3 loop alignment and tropism prediction using clonal data, clinical data and the 11/25 rule are shown in Fig. 1.

CD4 T cell counts at each interval were lower for patients with X4-tropic viruses and were significantly different when tropism was determined by geno 2 pheno $_{\text {[clinical }}$ ${ }_{20 \%]}$ (Fig. 2A, B). CD4 T cell counts and VLs according to different tropism prediction criteria are described in Table. CD4 T lymphocyte cell counts throughout the 48 weeks (Fig. 2C) and VLs (Fig. 2D) were comparable for patients infected with either GWGR or GPGR variants.

Taking into consideration the FPR values generated using geno2pheno ${ }_{\text {clinica } 20 \% 1}$ for each isolate from all cases (ITT analysis), the CD4 T cell gain showed a positive, significant correlation with FPR values at all observation weeks. This was observed by both linear regression $(p<0.0001)$ (Fig. 3) and the Spearman linear correlation $(p<0.01)$. A similar trend was also observed when only those completing week 48 of follow-up were evaluated ("as treated", data not shown). Baseline CD4 $\mathrm{T}$ cell counts and geno2pheno ${ }_{\text {[clonal20\%] }}$ FPR values were not significantly correlated with CD4 T cell gain ( $\mathrm{p}$ $=0.3$ and 0.2 , respectively).

\section{DISCUSSION}

This study evaluated the potential role of the molecular characteristics of the HIV-1 envelope on CD4 T lymphocyte gains in patients initiating their first HAART regimen from a structured cohort study performed to compare two first-line regimens. Viral tropism was determined by bioinformatics tools, using the geno2pheno algorithm with different criteria and inspecting the amino acid composition of the V3 loop of env (11/25 rule). The geno2pheno clinical option incorporated VL, CD4 T lymphocyte cell counts and percentages to generate an FPR value in which the predicted tropism was weighted by these parameters. Sequences were also evaluated using the MOTIVATE option, which is the most specific criterion for detection of X4-tropic viruses, as it uses a more stringent FPR cut-off. It is of note that both the MOTIVATE study and the European consensus recommend lower cut-offs of $5.75 \%$ and $10 \%$, respectively, when only three or more sequences of each individual are available. Therefore, a cut-off of 20\% was used for all analyses, as recommended by the European consensus for single sequences. The results exhibited some discordance in tropism interpretation when different algorithms were 


\begin{tabular}{|c|c|c|c|c|c|c|c|c|c|c|c|c|c|}
\hline$G R$ & 1 & 11 & & 15 & 16 & 17 & 18 & & 25 & 35 & $\begin{array}{c}\text { FPR } \\
\text { Clonal }\end{array}$ & $\begin{array}{c}\text { FPR } \\
\text { Clinical }\end{array}$ & $11 / 25$ \\
\hline 1169 & CTSPGTXTRX & $s$ & $\mathrm{MxI}$ & G & $\mathrm{P}$ & G & $\mathrm{R}$ & AXYAT - & D/A/E & I I GDXRXAHC & 6.8 & 0.4 & R5 \\
\hline 1170 & CTRXSNNTRR & $\mathrm{s}$ & VHL & G & $\mathrm{P}$ & G & $\mathrm{R}$ & $S F Y A T G$ & $\mathrm{E}$ & I I GD I RXAHC & 74.3 & 49.8 & R5 \\
\hline 1175 & C TRPNNNTRX & $\mathrm{s}$ & I H I & G & $\mathrm{P}$ & G & $\mathrm{R}$ & A FYATG & $\mathrm{E}$ & I I GK I RQAYC & 49 & 41.6 & R5 \\
\hline 1181 & C I RPNNNTRX & G & I H I & G & $\mathrm{P}$ & G & $\mathrm{R}$ & $A X Y A T G$ & D & I I GD I RKAHC & 25.6 & 9.3 & R5 \\
\hline 1187 & C TR P NNNTRK & $\mathrm{s}$ & $\mathrm{I} \mathrm{NL}$ & G & $\mathrm{P}$ & G & $\mathrm{R}$ & A I Y A T G & Q/E & $|X G D| R Q A H C$ & 17.3 & 1.5 & ( \\
\hline 1188 & CTRPNNNTRK & G & $\mathrm{IHI}$ & G & $\mathrm{P}$ & G & $\mathrm{R}$ & AFFAT- & D & I I GD IRXAHC & 62.5 & 75.2 & . \\
\hline 1191 & C I R PNNNTRK & $\mathrm{s}$ & V H L & G & $\mathrm{P}$ & G & $\mathrm{R}$ & AFYAT- & D & I I GD I RQAYC & 21.7 & 6.3 & 25 \\
\hline 1194 & C SRPNNNTRK & $\mathrm{s}$ & I HL & G & $\mathrm{P}$ & G & $\mathrm{R}$ & AFYATG & D & I I GD I RKAHC & 35.1 & 65.4 & \\
\hline 1195 & C TR P NNNTRK & $\mathrm{s}$ & $|\mathrm{P}|$ & G & $\mathrm{P}$ & G & $\mathrm{R}$ & A F Y A T G & D & I I GN I RQAHC & 89.1 & 43.4 & int \\
\hline 1201 & $C T R P G X N X R K$ & s & I HR & G & $\mathrm{P}$ & G & $\mathrm{R}$ & A FYATG & G & I I GD IXKAXC & 2.6 & 28.6 & \\
\hline 1203 & C TR PGNNTRX & s & I H I & G & $\mathrm{P}$ & G & $\mathrm{R}$ & A FYATG & $D / E$ & I I GN I RQAHC & 41.3 & 78.8 & 15 \\
\hline 1205 & C T R P NNNTRK & G & I LX & G & $\mathrm{P}$ & G & $\mathrm{R}$ & A F Y A T X & $\mathrm{s}$ & I I GX I RQAYC & 19.1 & 21.7 & 5 \\
\hline 1208 & C T R HNNNTRX & $\mathrm{s}$ & $\mid \mathrm{I} \times \mathrm{x}$ & G & $\mathrm{P}$ & G & $\mathrm{R}$ & A F Y A T G & D & I I GD I RQAHC & 47.6 & 89.6 & 25 \\
\hline \multicolumn{14}{|c|}{\begin{tabular}{l|l} 
GWGR &
\end{tabular}} \\
\hline 1167 & C TRPSNNTRX & $\mathrm{s}$ & $\mathrm{xH} \mathrm{M}$ & G & w & G & $\mathrm{R}$ & A F S A T G & $\mathrm{E}$ & I TGD I RRAHC & 2.9 & 9.2 & 15 \\
\hline 1168 & C TRHNNNTRK & G & I H I & G & w & G & $\mathrm{R}$ & TFYAT G & $\mathrm{E}$ & I I GD IRQSHC & 86.5 & 70.4 & 25 \\
\hline 1171 & C TRPGNNTRK & $\mathrm{s}$ & I H L & G & W & G & $\mathrm{R}$ & A F Y A T G & D & I I GD IRKAYC & 13 & 4.9 & 5 \\
\hline 1180 & C TRLSNNTRK & $\mathrm{s}$ & I H L & G & w & G & $\mathrm{R}$ & AFYATX & $\mathrm{E}$ & I XGDXXKAHC & 17 & 14.6 & 15 \\
\hline 1184 & C TRLSNNTRK & $\mathrm{s}$ & I H M & G & w & G & $\mathrm{R}$ & A FYT T G & $\mathrm{E}$ & I I GD I RXAHC & 42.6 & 26.2 & \\
\hline 1192 & C TRPNNNTRK & $\mathrm{s}$ & I H L & G & W & G & $\mathrm{R}$ & T L Y A T G & A & I I GD I R KAFC & 47 & 3.6 & 25 \\
\hline 1202 & CTRPXXNTRX & $\mathrm{s}$ & $|\mathrm{x} x|$ & G & W & G & R $P$ & $X L Y A X G$ & A & I I GD I RQAHC & 68.3 & 32.6 & 5 \\
\hline 1204 & C TRPGNNTRK & $\mathrm{s}$ & I H L & G & w & G & $\mathrm{R}$ & A F Y ATG & $\mathrm{E}$ & I VGD IXQAHC & 18.3 & 3.4 & 15 \\
\hline 1209 & CTRPSNNTRK & $\mathrm{s}$ & I H L & G & w & G & $\mathrm{R}$ & A F Y ATG & $\mathrm{E}$ & I TGD I RQAHC & 41.4 & 3.6 & \\
\hline \multicolumn{14}{|c|}{ 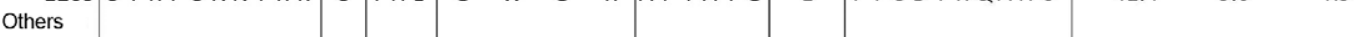 } \\
\hline 1176 & CTRPNNNTRK & G & I H L & G & $\mathrm{F}$ & G & Q & A L Y A T G & G & I VGD I RQAHC & 73.4 & 9.6 & \\
\hline 1179 & C TRPSNNTRK & G & I H L & G & $\mathrm{F}$ & G & $\mathrm{R}$ & T I FAT G & $\mathrm{E}$ & $|X G D| R Q A H C$ & 23 & 16.9 & 5 \\
\hline 1189 & C TRPNNNTRK & G & I H I & G & $\mathrm{P}$ & G & A & A F Y T T - & $\mathrm{N}$ & I VGD I RRAHC & 20.9 & 16.2 & 15 \\
\hline 1172 & CTRPNNNTRK & G & I H I & G & $\mathrm{P}$ & G & $\mathrm{K}$ & A FYT A - & D & I I GD I RQAHC & 15 & 10.6 & R5 \\
\hline 1190 & C TRPNNNTRK & G & I H I & G & $\mathrm{P}$ & G & $\mathrm{K}$ & VFYT T - & D & I I GD I RQAHC & 11.7 & 1.2 & \\
\hline 1185 & C TRPGN & G & I H I & G & $\mathrm{P}$ & G & $\mathrm{Q}$ & A I Y A T G & $\mathrm{R}$ & I XGD I RXAHC & 9.9 & 10.6 & 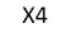 \\
\hline 1207 & CTRPNNNTRX & G & I H L & G & $\mathrm{P}$ & G & $\mathrm{s}$ & $T L Y A T G$ & E & I VGNXRQAHC & 92.6 & 42.8 & 15 \\
\hline 1177 & CTRPNNNTRK & $\mathrm{S} / \mathrm{G}$ & $x \times 1$ & G & $\mathrm{P}$ & $G R$ & $\mathrm{R} / \mathrm{G}$ & AWYATX & K/E & $X \vee G D \mid X X A Y C$ & 5.7 & 3.6 & $\mathrm{x} 4$ \\
\hline 1178 & C TRPGNYTSK & G & I R F & G & $\mathrm{P}$ & $G \quad K$ & $\mathrm{~K} / \mathrm{Q}$ & AFYAXG & $\mathbf{R}$ & I I GD I RQAHC & 1.8 & 1.2 & $<4$ \\
\hline 1183 & C TRPGNNTSK & G & $\mathrm{IHX}$ & G & $\mathrm{P}$ & $G R$ & $\mathrm{R} / \mathrm{G}$ & AFYATX & $R / A / S$ & I I GD I RQAHC & 17 & 30.6 & $\times 4$ \\
\hline 1197 & CTRPNNNTRK & $\mathrm{s}$ & I H M & G & $\mathrm{P}$ & $G \quad R$ & $\mathrm{R} / \mathrm{K}$ & A F Y T T G & $\mathrm{s}$ & I I GD I RQAHC & 33.9 & 15 & R5 \\
\hline 1200 & C TRPNNNTRK & G & I H L & G & $\mathrm{P}$ & $G \quad R$ & $R / K$ & TMY A T G & A & I I GD IRKAYC & 44.5 & 3.6 & R5 \\
\hline 1182 & C T R P SNNTRK & $\mathrm{s}$ & $1 \times \mathrm{M}$ & A/G & w & G & $\mathrm{R}$ & $X X Y A T G$ & D & I I GD I RQAHC & 71.7 & 25.3 & R5 \\
\hline 1199 & CTRPNNNTRK & G & $I Q M$ & A & w & G & $\mathrm{R}$ & TFYATG & E & I I GD I RQAHC & 93.5 & 57.4 & $r$ \\
\hline 1173 & C TRPGNNTRK & G & $\mathrm{XHL}$ & G & W & $\mathrm{G} / \mathrm{R}$ & $\mathrm{R}$ & $T F X X T X$ & $\mathrm{~K}$ & I IGD IRXAHC & 2.7 & 0.8 & 44 \\
\hline 1174 & C TRPNNNTGK & $\mathbf{R}$ & I H M & G & W c & $\mathrm{G} / \mathrm{R}$ & $\mathrm{R}$ & XFYATX & G & I I GD IXQAHC & 1.7 & 0 & \\
\hline 1193 & C TR P N N NTRR & $\mathrm{s}$ & $\mathrm{I} \mathrm{HI}$ & G & W & $\mathrm{R}$ & $\mathrm{R}$ & T F Y A A G & $\mathrm{E}$ & I I RD IRKAYC & 70.1 & 1.3 & R5 \\
\hline 1198 & C TRPNNNTRX & $\mathrm{S} / \mathrm{G}$ & $|x|$ & G & $P / L$ & $\mathrm{x}$ & $\mathrm{R}$ & AFYT T G & $\mathrm{E}$ & I I GD I RQAXC & 30 & 46.1 & R5 \\
\hline 1186 & CTRPNNNTRK & s & I H M & G & $\mathrm{W} / \mathrm{L}$ & G & $\mathrm{R}$ & A F Y ATG & E & I I GX I RQAXC & 31.3 & 43.8 & 85 \\
\hline 1196 & CTRPNNNTRK & $\mathrm{S} / \mathrm{G}$ & I HL & G & $F / L$ & G & $\mathrm{R}$ & A F Y XTG & $D / Q$ & $|I| G X \mid R Q A X C$ & 15.7 & 10.3 & \\
\hline
\end{tabular}

Fig. 1: amino acid alignment of V3 loop. Selected positions represent codons 11/25 and V3 tip codons 15-18. Bold characters represent basic amino acids at positions 11 and 25 (left). First and second columns show false-positive rate (FPR) values obtained through geno2pheno clonal and clinical options. Third column represents tropism prediction according to rule 11/25 (right).

used. Interestingly, most patients had advanced disease at the beginning of treatment, with a mean CD4 T cell count of 119 cells $/ \mathrm{mm}^{3}$; this could explain the tropism disparities when clinical data were incorporated.

To assess the impact of viral tropism on clinical evolution, both VL suppression and CD4 T cell recovery were followed-up for one year. Treatment, tDRM, V3 loop motif and viral tropism did not impact VL suppression. Lower numbers of CD4 T cells were observed at both the baseline and the follow-up weeks in patients infected with X4 virus using both clinical and clonal algorithms. Furthermore, CD4 T cell numbers were significantly lower in patients harbouring $\mathrm{X} 4$ variants when prediction used the geno2pheno ${ }_{[\mathrm{clinical20 \%}}$ option (Fig. 2A). The use of baseline CD4 T cell counts on the clinical algorithm could be responsible for this stronger association. However, baseline CD4 T cell counts did not show a correlation with CD4 $\mathrm{T}$ cell gain $(\mathrm{p}=0.3)$. Although CD4 T cell counts of R5infected patients remained higher at most determinations, CD4 T cell recovery was similar for both groups. In other words, despite the higher CD4 T cell counts in R5-infected patients, the response to HAART was comparable for both R5 and X4-infected individuals.

Values of FPR as continuous variables were also evaluated to assess its association to CD4 T cell gain. Using the geno2pheno ${ }_{\text {[clinical] }}$ option, envelope sequences FPR values exhibited a strong correlation with $\mathrm{CD} 4 \mathrm{~T}$ cell gain $(p<0.0001)$ (Fig. 3). Again, although CD4 T cell count is used in this model to determine geno2pheno ${ }_{\text {[clinical] }} \mathrm{FPR}$, neither CD4 T cell baseline counts $(\mathrm{p}=0.3)$ nor the clonal FPR $(p=0.2)$ per se revealed a significant correlation with CD4 T cell gain. This suggests that FPR generated by the geno2pheno clinical algorithm may constitute a potential predictor for CD4 T cell gain after therapy. The usefulness of this option has also been proposed in previous studies (Prosperi et al 2010).

Neither the GWGR nor the GPGR V3 loop motif had an association with CD4 $\mathrm{T}$ cell gain, despite the fact that CD4 $\mathrm{T}$ cell numbers were slightly higher at baseline in the former group (Fig. 2C). An important 

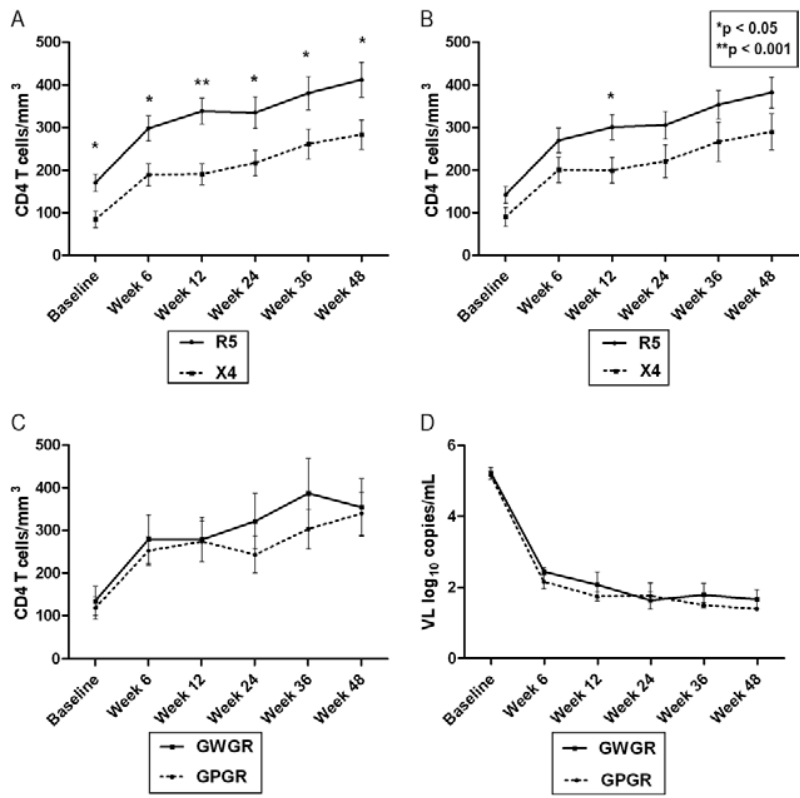

Fig. 2: CD4 T cell counts at baseline and at each week of observation (6-48 weeks) according to predicted tropism using geno2pheno $_{\text {[clinical } 20 \%]}$ cut-off (A), geno2pheno ${ }_{\text {[clonal120\%] }}$ cut-off (B), V3 loop motifs (GWGR and GPGR) (C) and viral load (VL) suppression according to V3 loop motifs (D).

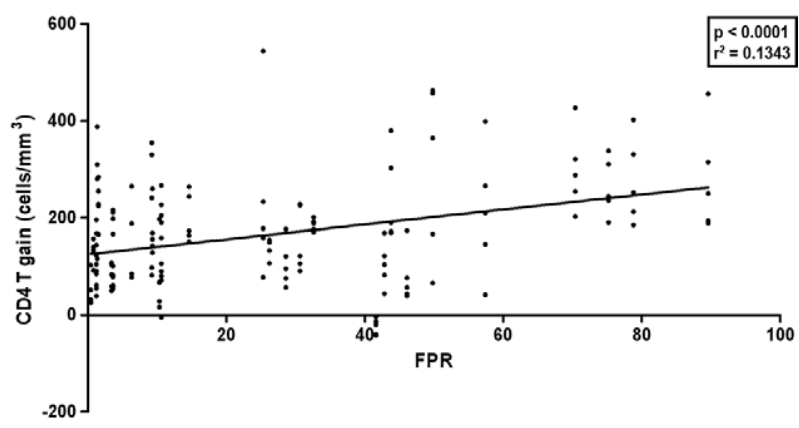

Fig. 3: linear regression of $\mathrm{CD} 4 \mathrm{~T}$ cell gain plotted according to geno2 pheno $_{\text {[clinical] }}$ option false-positive rate (FPR) obtained from baseline samples (intention to treat, $\mathrm{p}<0.0001$ ). Replicates represent CD4 $\mathrm{T}$ cell gain at each observation week.

prevalence of X4 variants in GWGR viruses was observed using both the geno2pheno ${ }_{[\text {lonal20\%] }}(44 \%)$ and the geno2 pheno $_{[\text {[clinical20\%] }}(67 \%)$ algorithms, contrary to previous studies, which suggested that GWGR viruses are rarely X4 (da Silva 2006, Leal et al. 2008). This difference could be due to the algorithm used, as most studies use the 11/25 rule as a prediction criterion. To clarify this matter, the presence of basic amino acids at positions 11 and 25 was evaluated in our sequences; none of the GWGR or GPGR isolates had basic amino acids at these codons. Interestingly, three V3 tip sequences of non-GWGR tryptophan-harbouring viruses, classified here as "other motifs", were predicted as
$\mathrm{X} 4$. These viruses had a $\mathrm{G} \rightarrow \mathrm{R}$ polymorphism in the $\mathrm{V} 3$ loop tip at position $17\left({ }_{15} \mathrm{GWRR}_{18}\right)$, two had basic amino acids at codons 11 or 25 and one had a $\mathrm{G} \rightarrow \mathrm{R}$ substitution at position 28, a highly conserved codon (Leal et al. 2008, Franca et al. 2011). GWRR seems to be a very rare motif previously documented in the state of Rio de Janeiro, Brazil and Paraguay and its biological relevance is yet to be understood (Cabello et al. 1995, Tanuri et al. 1999)

The small number of samples in this study limits the strength of these observations and it is conceivable that these outcomes may be different if a larger cohort was sampled and a longer follow-up period was employed. Further studies may provide support for the use of geno2pheno ${ }_{\text {[clinical] }}$ analysis of V3 envelope sequences as a surrogate marker for CD4 T cell gain after ART.

\section{ACKNOWLEDGEMENTS}

To the volunteers and the staff of the STI clinic involved in the study, and to Gabriela Ribeiro Santos, for the English review.

\section{REFERENCES}

Cabello A, Cabral M, Vera ME, Kiefer R, Azorero RM, Eberle J 1995. Analysis of the V3 loop sequences from 10 HIV type 1-infected AIDS patients from Paraguay. AIDS Res Hum Retroviruses 11: 1135-1137.

Casseb J, Komninakis SCV, Abdalla L, Brigido LFM, Rodrigues R, Araujo F 2002. HIV disease progression: is the Brazilian variant subtype B' (GWGR motif) less pathogenic than US/European subtype B (GPGR)? Inter J Infect Dis 6: 64-69.

Casseb J, Montanheiro P, Komninakis S, Brito A, Duarte AJS 2004. Human immunodeficiency virus type 1 Brazilian subtype B variant showed an increasing avidity of the anti-V3 antibodies over time compared to the subtype B US/European strain in São Paulo, Brazil. Mem Inst Oswaldo Cruz 99: 69-71.

da Silva J 2006. Site-specific amino acid frequency, fitness and the mutational landscape model of adaptation in human immunodeficiency virus type 1. Genetics 174: 1689-1694.

de Brito A, Komninakis SCV, Novoa P, de Oliveira RM, Fonseca LAM, Duarte AJS 2006. Women infected with HIV type 1 Brazilian variant, subtype B (B'-GWGR motif) have slower progression to AIDS compared with patients infected with subtype B (B-GPGR motif). Clin Infect Dis 43: 1476-1481.

Ferreira JLP, Thomaz M, Rodrigues R, Harrad D, Oliveira CM, Oliveira CAF, Batista JPGB, Ito TS, Brigido LFMB 2008. Molecular characterizations of newly identified HIV-1 infections in $\mathrm{Cu}-$ ritiba, Brazil: preponderance of clade $\mathrm{C}$ among males with recent infections. Mem Inst Oswaldo Cruz 103: 800-808.

Franca RFO, Castro-Jorge LA, Neto RJP, Jorge DMM, Lima DM, Colares JKB 2011. Genotypic characteristics of HIV type 1 based on gp120 hypervariable region 3 of isolates from southern Brazil. Aids Res Hum Retroviruses 27: 903-909.

Goetz MB, Leduc R, Kostman JR, Labriola AM, Lie Y, Weidler J 2009. Relationship between HIV coreceptor tropism and disease progression in persons with untreated chronic HIV infection. J Acquir Immune Defic Syndr 50: 259-266.

Leal E, Silva WP, Sucupira MC, Janini LM, Diaz RS 2008. Molecular and structural characterization of HIV-1 subtype B Brazilian isolates with GWGR tetramer at the tip of the V3-loop. Virology 381: 222-229. 
Pinto ME, Schrago CG, Miranda AB, Russo CAM 2008. A molecular study on the evolution of a subtype B variant frequently found in Brazil. Genet Mol Res 7: 1031-1044.

Potts KE, Kalish ML, Lott T, Orloff G, Luo C, Bernard M, the Brazilian Collaborative AIDS Research Group 1993. Genetic heterogeneity of the V3 region of the HIV-1 envelope glycoprotein in Brazil. AIDS 7: 1191-1197.

Prosperi M, Bracciale L, Fabbiani M 2010. Comparative determination of HIV-1 co-receptor tropism by enhanced sensitivity trofile, gp120 V3-loop RNA and DNA genotyping. Retrovirology 7: 56.

Richman DD, Bozzette SA 2004. The impact of the syncytium inducing phenotype of human immunodeficiency virus on disease progression. J Infect Dis 169: 968-974.

Santoro-Lopes G, Harrison LH, Tavares MD, Xexéo A, dos Santos ACE, Schechter M 2000. HIV disease progression and V3 sero- types in Brazil: is B different from B-Br? AIDS Res Hum Retroviruses 16: 953-958.

Shioda T, Levy JA, Cheng-Mayer C 1991. Macrophage and T cellline tropisms of HIV-1 are determined by specific regions of the envelope gp120 gene. Nature 349: 167-169.

Sing T, Low AJ, Beerenwinkel N, Sander O, Cheung PK, Domingues F, Büch J, Däumer M, Kaiser R, Lengauer T, Harrigan PR 2007. Predicting HIV co-receptor usage based on genetic and clinical covariates. Antivir Ther 12: 1097-1106.

Tanuri A, Swanson P, Devare S, Berro OJ, Savedra A, Costa LJ 1999. HIV-1 subtypes among blood donors from Rio de Janeiro, Brazil. J Acquir Immune Defic Syndr Hum Retrovirol 20: 60-66.

Vandekerckhove LPR, Wensing AMJ, Kaiser R, Brun-Vézinet F, Clotet B, de Luca A, the European Consensus Group on Clinical Management of Tropism Testing 2011. European guidelines on the clinical management of HIV-1 tropism testing. Available from: thelancet.com/infection. 